EPJ B manuscript No.

(will be inserted by the editor)

\title{
Anisotropy and field-dependence of the spin-density-wave dynamics in the quasi one-dimensional conductor (TMTSF) ${ }_{2} \mathbf{P F}_{6}$
}

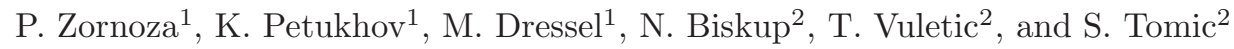 \\ 1 1. Physikalisches Institut, Universität Stuttgart, Pfaffenwaldring 57, D-70550 Stuttgart, Germany \\ 2 Institut za fiziku, P.O. Box 304, HR-10001 Zagreb, Croatia
}

Received: October 31, 2018/ Revised version:

\begin{abstract}
The anisotropic and non-linear transport properties of the quasi one-dimensional organic conductor (TMTSF $)_{2} \mathrm{PF}_{6}$ have been studied by dc, radiofrequency, and microwave methods. Microwave experiments along all three axes reveal that collective transport, which is considered to be the fingerprint of the spin-density-wave condensate, also occurs in the perpendicular $b^{\prime}$ direction. The pinned mode resonance is present in the $a$ and $b^{\prime}$-axes response, but not along the least conducting $c^{*}$ direction. The ac-field threshold, above which the spin-density-wave response is non-linear, strongly decreases as the temperature drops below $4 \mathrm{~K}$. With increasing strength of the microwave electric field and of the radiofrequency signal, the pinned mode and the screened phason loss-peak shift to lower frequencies. In the non-linear regime, in addition to the phason relaxation mode with Arrhenius-like resistive decay, an additional mode with very long and temperature-independent relaxation time appears below $4 \mathrm{~K}$. We attribute the new process to short-wavelength spin-density-wave excitations associated with discommensurations in a random commensurate $N=4$ domain structure.
\end{abstract}

PACS. 72.15.Nj Collective modes (e.g., in one-dimensional conductors) - 75.30.Fv Spin-density waves 74.70.Kn Organic superconductors

\section{Introduction}

The spin-density-wave (SDW) ground state of one-dimensional conductors attracts considerable attention even after two decades of intensive research. Most experimental studies have been performed on the quasi one-dimensional organic Bechgaard salt di-(tetramethyltetraselenafulvalene)hexaflourophosphate, denoted as (TMTSF) ${ }_{2} \mathrm{PF}_{6}$, which soon became the model compound of this phenomenon. Since a large number of results have been accumulated over the years, the SDW state of (TMTSF) ${ }_{2} \mathrm{PF}_{6}$ can be considered as understood to a fair extent. Nevertheless, there are certain issues which still wait for a clarification 1. 2,3 .

At $T_{\mathrm{SDW}}=12 \mathrm{~K},(\mathrm{TMTSF})_{2} \mathrm{PF}_{6}$ undergoes a metalinsulator transition below which a thermally activated transport is observed. The insulating SDW ground state is a consequence of the instability of the quasi one-dimensional Fermi surface. In particular NMR experiments reveal that the nesting vector in (TMTSF $)_{2} \mathrm{PF}_{6}$ is incommensurate: $\mathbf{Q}=\left[0.5 a^{*},(0.24 \pm 0.03) b^{*},(-0.06 \pm 0.20) c^{*}\right]$ [4.

In the SDW state the optical conductivity develops an absorption edge in the infrared spectral range due to the opening of the single-particle gap at the Fermi energy. While it turned out to be extremely difficult to observe the gap in the direction along the chains because

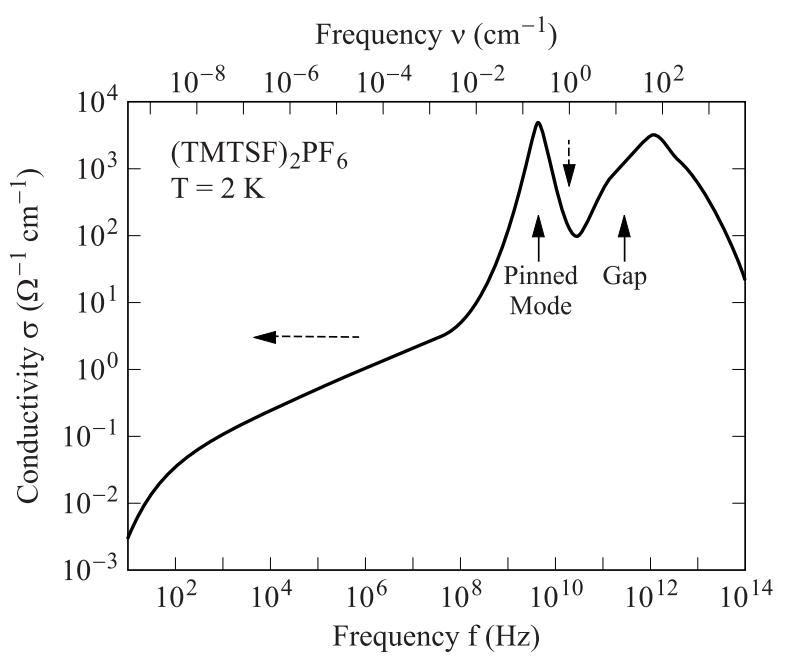

Fig. 1. Sketch of the frequency dependent SDW conductivity composed using various sets of data along the chain axis of (TMTSF $)_{2} \mathrm{PF}_{6}$. The solid arrows indicate the position of single particle gap and the pinned mode resonance in the microwave frequency range (after [7). The dashed vertical and horizontal arrows depict the frequency range of the investigations presented in this work. 
P. Zornoza et al.: Anisotropic and field-dependent pinning of SDW

the spectral range is strongly reduced in this range of frequency (so-called clean limit), optical experiments along the perpendicular direction 5] unambiguously proved the development of the SDW gap at $2 \Delta /(h c)=70 \mathrm{~cm}^{-1}$. The size of the SDW gap detected in dc-limit is much smaller (approximately $30 \mathrm{~cm}^{-1}$ ), probably due to imperfections inducing in-gap states or due to the dispersion of the energy bands in quasi one-dimensional compounds 6]. A so-called pinned mode resonance is found around $5 \mathrm{GHz}$; it can be attributed to the collective response of the condensate pinned to lattice imperfections [7]. It contains only a very small fraction of the spectral weight missing after the collapse of the Drude response upon entering the insulating SDW state. At even lower frequencies (in the range of $\mathrm{MHz}, \mathrm{kHz}$ and even below, depending on temperature) internal deformations and screening by the conduction electrons lead to a broad increase of the conductivity and corresponding broad relaxational behavior. The overall behavior of the optical response is plotted in Fig. 1.

A large number of investigations have been dedicated to the non-linear transport of the SDW ground state 7. 8.9. In very low fields $\left(E \ll E_{T}\right)$, as expected for a typical semiconductor, Ohmic behavior is observed along the chains. However in electric fields which exceed a characteristic threshold field $E_{T}$, a contribution to the conductivity due to the sliding of the SDW was expected and experimentally found. $E_{T}$ is typically in the $3-8 \mathrm{mV} / \mathrm{cm}$ range in nominally pure specimens, with a factor of two increase of the conductivity in fields which are several times larger than $E_{T}$. The existence of both narrow-band noise 10 , 11] and Shapiro interferences [1] above $E_{T}$ provide very strong indirect evidence that the nonlinearity observed is associated with a depinning of the SDW, however the large motional narrowing of the proton lineshape seen above $E_{T}$ 12 is by far the most conclusive proof. As expected for a SDW which is pinned to impurities, the size of $E_{T}$ has been correlated to the concentration of lattice defects induced by X-ray irradiation [8] as well as to the random disorder introduced by the alloying at the anion sites.

In this Communication the issue of the anisotropic and field dependent response of the collective mode is addressed. To our knowledge, the non-linear conduction and the pinned mode resonance - fingerprints of the collective response - have only been investigated along the chain direction. Also not much is known about the radiofrequency and microwave response with increasing amplitude of the driving ac field. Finally, the internal structure of the SDW and the origin of anomalies observed in the spin-lattice relaxation rate, specific heat and magneto-resistance, have not been consistently clarified yet [13,14,15].

\section{Experimental Details}

Single crystals of the Bechgaard salt (TMTSF) ${ }_{2} \mathrm{PF}_{6}$ were grown by electrochemical methods as described in Ref. 16. After several months we were able to harvest needle-shaped to flake-like single crystals of several millimeters in length ( $a$ axis) and a considerable width ( $b^{\prime}$-direction) up to $2 \mathrm{~mm}$.
Here $a$ indicates the stacking direction of TMTSF molecules. Due the triclinic symmetry, $b^{\prime}$ denotes the projection of the $b$ axis perpendicular to $a$, and $c^{*}$ is normal to the $a b$ plane. The crystals were characterized by dc resistivity measurements. Along the $a$-axis the experiments were performed on needle-shaped samples with a typical dimension of $2 \mathrm{~mm} \times 0.5 \mathrm{~mm} \times 0.1 \mathrm{~mm}$ along the $a, b^{\prime}$, and $c^{*}$ axes, respectively. The results on the $b^{\prime}$-axis conductivity were obtained on a narrow slice cut from a thick crystal perpendicular to the needle axis; the typical dimensions of so-made samples were $a \times b^{\prime} \times c^{*}=0.2 \mathrm{~mm} \times 1.3 \mathrm{~mm} \times 0.3 \mathrm{~mm}$. Due to our advances in achieving large sample geometry, we were able to measure $b^{\prime}$-axis resistivity for the first time with basically no influence of the $a$ and $c^{*}$ contributions and using standard four-probe technique to eliminate the contact resistance. Also for the $c^{*}$-axis transport, we were able to apply four contacts, two on each side of the crystal. The contacts were made by evaporating gold pads on the crystal, then $25 \mu \mathrm{m}$ gold wires were pasted on each pad with a small amount of silver or carbon paint. The (TMTSF $)_{2} \mathrm{PF}_{6}$ samples were slowly cooled down to avoid cracks and ensure a thermal equilibrium.

The real and imaginary parts of the conductance, $G(\omega)$ and $B(\omega)$, were measured employing a HP4284A impedance analyzer $(f=20 \mathrm{~Hz}$ to $1 \mathrm{MHz})$ in the two-probe configuration. Three samples with lengths from 0.21 to $0.34 \mathrm{~cm}$ and cross-sections in the range of $2.6 \cdot 10^{-5}$ to $10^{-4} \mathrm{~cm}^{2}$ were studied in the temperature range between $1.5 \mathrm{~K}$ and $5 \mathrm{~K}$; they all exhibited qualitatively the same behavior. The data were taken by sweeping the frequency at a fixed temperature. Both cooling and warming cycles were conducted and no hysteresis was observed. In order to study the non-linear transport, an ac signal amplitude $V_{S}$ was applied in the range between 0.07 and 1 of the dc threshold voltage $V_{T}$ for non-linear conductivity; which at $T=4.2 \mathrm{~K}$ was in the range from $V_{T}=2 \mathrm{mV}$ to $4 \mathrm{mV}$. As the smallest ac voltage delivered by the HP4284A is $5 \mathrm{mV}$, a homemade electronic circuit was utilized to reduce the voltage effective on the sample to the desired level. Dielectric functions were extracted from the conductivity using the relations $\varepsilon^{\prime}(\omega)=B(\omega) / \omega$ and $\varepsilon^{\prime \prime}(\omega)=\left[G(\omega)-G_{0}\right] / \omega$. The observed dielectric response can be well fitted by the phenomenological Havriliak-Negami (HN) function widely used to describe relaxation processes in disordered systems

$$
\varepsilon(\omega)-\varepsilon_{\mathrm{HF}}=\frac{\Delta \varepsilon}{1+\left(i \omega \tau_{0}\right)^{1-\alpha}}
$$

Here $\Delta \varepsilon=\varepsilon_{0}-\varepsilon_{\mathrm{HF}}$ is the relaxation strength, and $\varepsilon_{0}$ and $\varepsilon_{\mathrm{HF}}$ are the static and the high frequency dielectric constant, respectively; $\tau_{0}$ denotes the mean relaxation time and $1-\alpha$ is the shape parameter which describes the symmetric broadening of the relaxation time distribution function. More details on low-frequency dielectric spectroscopy and the data analysis can be found in Ref. [17.

In order to measure the microwave conductivity the crystals were placed onto a quartz substrate and positioned in the maximum of the electric field of different cylindrical copper cavities, resonating in the $\mathrm{TE}_{011}$ mode at $24,33.5 \mathrm{GHz}$ and $60 \mathrm{GHz}$, respectively. Along the $a$ direction naturally grown needles could be used (typical 


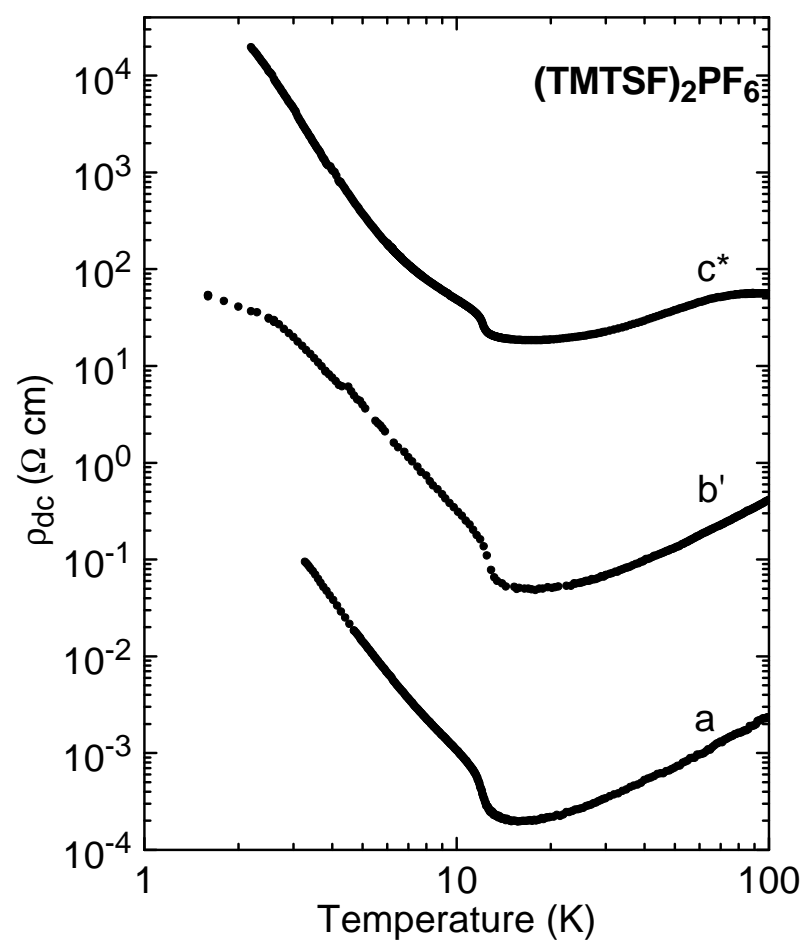

Fig. 2. Temperature dependence of the dc resistivity of (TMTSF $)_{2} \mathrm{PF}_{6}$ single crystals along the $a, b^{\prime}$, and $c^{*}$ crystallographic axes.

dimensions of $1 \mathrm{~mm} \times 0.2 \mathrm{~mm} \times 0.2 \mathrm{~mm})$ since this geometry is best for precise microwave measurements. To measure in $b^{\prime}$ direction, we cut a slice $\left(a \times b^{\prime} \times c^{*}=\right.$ $0.2 \mathrm{~mm} \times 1.2 \mathrm{~mm} \times 0.2 \mathrm{~mm}$ ) from a thick single crystal. In order to perform microwave experiments along the $c^{*}$ axis, a crystal was chopped into several pieces (approximately cubes of $0.2 \mathrm{~mm}$ corner size) and arranged up to four as a mosaic in such a way that a needle-shaped sample of about $(0.2 \times 0.2 \times 0.8) \mathrm{mm}^{3}$ was obtained. By recording the center frequency and the halfwidth of the resonance curve as a function of temperature $(1.5 \mathrm{~K}<T<300 \mathrm{~K})$ and comparing them to the corresponding parameters of an empty cavity, the complex electrodynamic properties of the sample, like the conductivity and the dielectric constant, can be determined via cavity perturbation theory; further details on microwave measurements and the data analysis are summarized in 16] 18, 19]. Using a microwave attenuator in the waveguide line between Gunn diode and the cavity allows us to reduce the power by $-18 \mathrm{~dB}$.

\section{Results}

The temperature dependence of the dc resistivity is displayed in Fig.2 in double logarithmic representation where the SDW transition at $T_{\mathrm{SDW}}=12 \mathrm{~K}$ is clearly seen in all three directions, $a, b^{\prime}$, and $c^{*}$. This is well understood since in this compound the single-particle gap opens over the entire Fermi surface. At lower temperatures the resistivity increases steadily with some exponential temperature dependence $\rho(T) \propto \exp \{\Delta / T\}$ due to excitations across

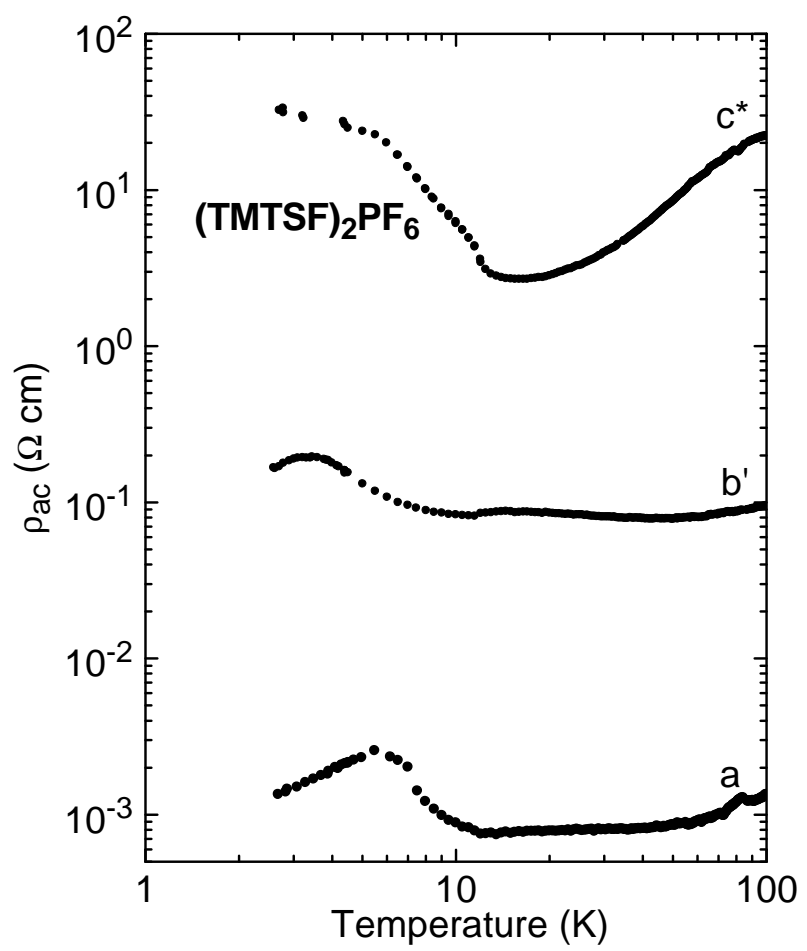

Fig. 3. Temperature dependence of the microwave resistivity of (TMTSF $)_{2} \mathrm{PF}_{6}$ along the $a, b^{\prime}$, and $c^{*}$ crystallographic axes, measured at $33.5 \mathrm{GHz}$.

the energy gap ranging from $\Delta \approx 20 \mathrm{~K}$ to $27 \mathrm{~K}[20$. The results are in excellent agreement with the predictions by the mean-field theory $\left(\Delta_{0}=1.76 k_{B} T_{\mathrm{SDW}}=21 \mathrm{~K}\right)$ and previous findings 3 .

The results obtained for the microwave response at $33.5 \mathrm{GHz}$ are plotted in Fig. 3 The SDW transition at $T_{\mathrm{SDW}}=12 \mathrm{~K}$ is again present in all orientations. In contrast to the dc response, however, for lowering the temperature the microwave resistivity increases monotonously below $T_{\text {SDW }}$ only in the $c^{*}$ direction. For the electric field parallel to the chains and along the $b^{\prime}$ direction, the behavior is more complicated; the resistivity saturates below approximately $4 \mathrm{~K}$ and in some cases even a maximum in $\rho_{a c}(T)$ is reached around $T \approx 4 \mathrm{~K}$ below which the resistivity decreases again.

\subsection{Anisotropic Response of the SDW Condensate}

In the temperature range slightly below $T_{\mathrm{SDw}}$, but still for $T>4 \mathrm{~K}, \rho(T)$ may be described by an activated behavior, as depicted in Fig. 0 [21. The activation energy along the $a$ and $b^{\prime}$ axes ( $\Delta \approx 5.9 \mathrm{~K}$ and $6.0 \mathrm{~K}$, respectively) is much smaller compared to the dc behavior, while for the $c^{*}$ orientation the results at microwave frequencies perfectly agree with the dc profile: $\Delta=20.7 \mathrm{~K}$. 


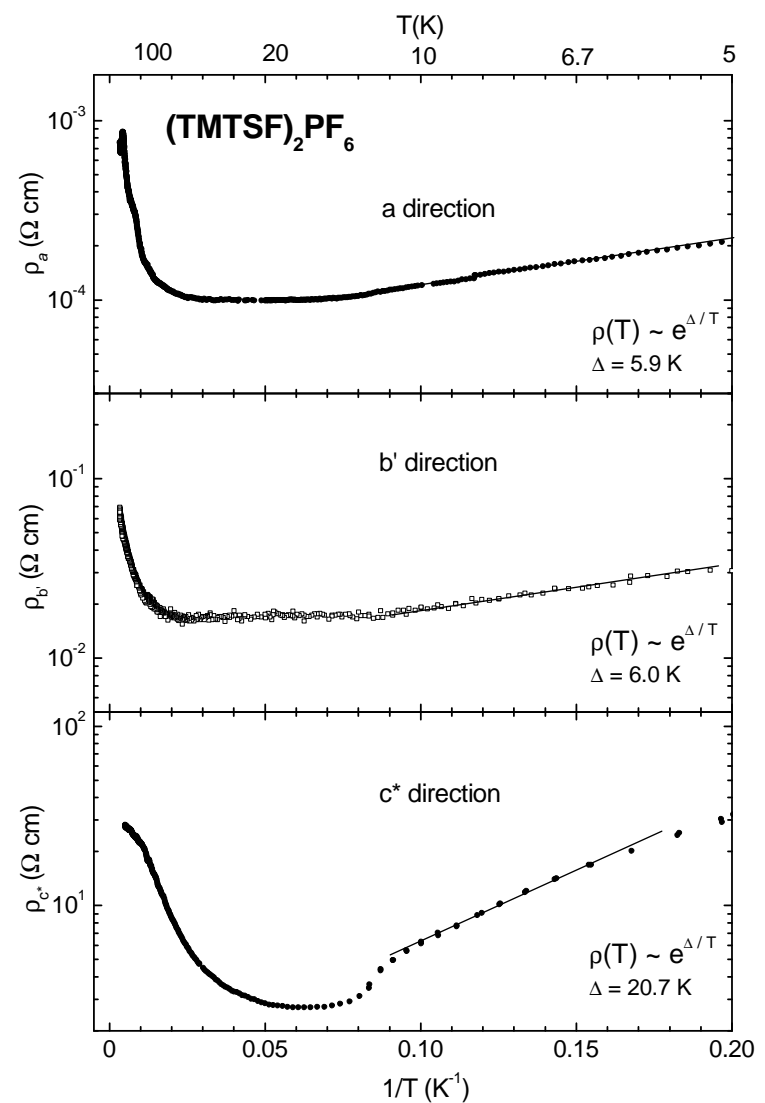

Fig. 4. Arrhenius plot of the microwave resistivity of (TM$\mathrm{TSF})_{2} \mathrm{PF}_{6}$ along the $a, b^{\prime}$ and $c^{*}$ directions measured at $33.5 \mathrm{GHz}$

\subsection{Field Dependent Response of the SDW Condensate}

Even more surprising is the decreasing resistivity for temperatures $T<4 \mathrm{~K}$. Although the actual shape, its temperature dependence and magnitude varies from sample to sample, the non-monotonous temperature dependence and overall behavior is robust. Up to six samples of different batches have been studied for each orientation leading to very similar findings. In fact, this puzzling behavior was occasionally reported by different groups $\left[\begin{array}{l|l|l|l|l|}\hline & 22 & 23 & 24 & 25 \\ \hline\end{array}\right.$ over the years, unfortunately without providing any mean-

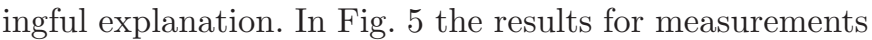
conducted at approximately $9 \mathrm{GHz}$ are compiled.

Donovan et al. 7 also investigated the microwave response at different frequencies from 3 to $150 \mathrm{GHz}$, and found that the deviations from the dc transport become less when going to higher-frequency resonance cavities [26]. Note, however, that the output power of the microwave source and thus the electric field strength inside the cavity strongly decreases with higher frequencies. It is also interesting to note, that the effect is less pronounced in those cases where the sample is located in the maximum of the magnetic field compared to measurements in the electric field maximum of the cavities. The present work tries to disentangle the influence of frequency and field strength.

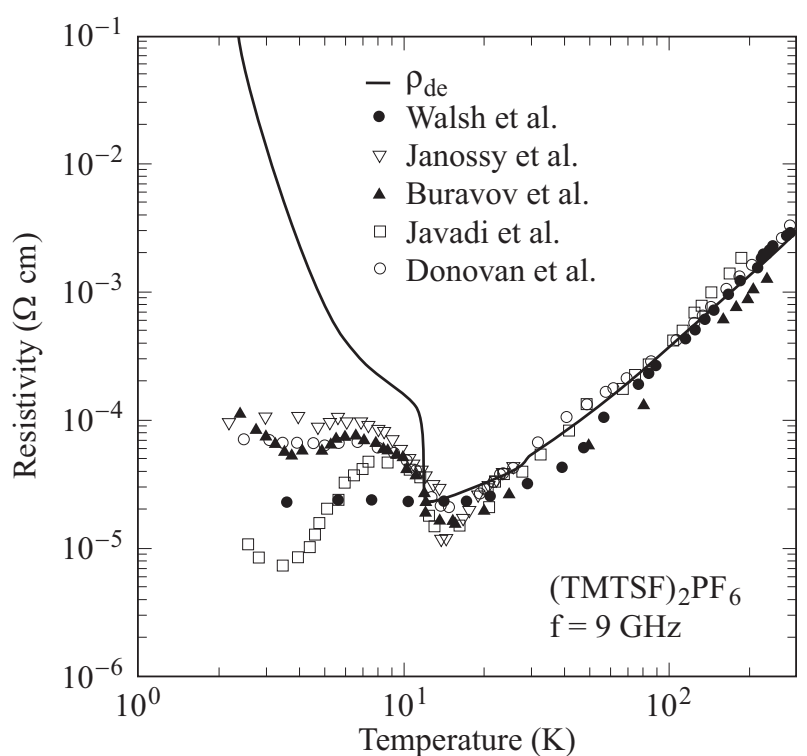

Fig. 5. Comparison of the temperature dependent microwave resistivity of (TMTSF) ${ }_{2} \mathrm{PF}_{6}$ along the $a$ axis reported by different groups. Data taken from Walsh et al. 22, Janossy et al. 23, Buravov et al. 24, Javadi et al. 25, and Donovan et al. 7]. For better comparison the data have been normalized to the $100 \mathrm{~K}$ dc value.

In order to further explore the non-monotonous behavior of $\rho_{a c}(T)$, we performed the cavity perturbation experiments along the $a$ and $b^{\prime}$ orientation using different microwave power, i.e. as a function of the electric field strength inside the cavity. The output power of the Gunn oscillator was attenuated from 0 to $-18 \mathrm{~dB}$. The power $P_{\text {input }}$ coupled into the cavity varies from $120 \mu \mathrm{W}$ to $2.5 \mu \mathrm{W}$, resulting in a reduction of the average electric field $E \propto \sqrt{P_{\text {input }}}$ from $54 \mathrm{mV} / \mathrm{cm}$ to $8 \mathrm{mV} / \mathrm{cm}$; in the antinode the field strength is larger by a factor of two. Note, however, that the absolute values only give a rough estime (within a factor of 5) due to uncertainties in the coupling to the cavity and losses in the waveguides. While Walsh et al. 22 estimate $1 \mathrm{mV} / \mathrm{cm}$ in their cavity, Buravov et al. 24 give $30 \mathrm{mV} / \mathrm{cm}$ for their $9 \mathrm{GHz}$ measurement. The latter group observed no change of the response when the electric field is reduced by a factor of 4 . In Fig. [6 the microwave resistivity is plotted as a function of inverse temperature for different values of the microwave power 21]. Reducing the power leads to a sharper increase below $T_{\text {SDW }}$ and the maximum in $\rho(T)$ shifts to higher temperature; the drop of the low-temperature resistivity becomes even more pronounced. This behavior is robust and was confirmed for different samples. Although this sort of field dependence is more or less observed for both orientations, along the $a$ and $b^{\prime}$ directions, due to the higher resistivity, the experiments are more precise for $E \| b^{\prime}$ and can cover a larger range of applied microwave power.

In Fig. 7 the power dependence of the resistivity $\rho_{a}$ and $\rho_{b^{\prime}}$ is plotted for a fixed temperature $T=2.5 \mathrm{~K}$. In both cases a linear dependence on the input power is found with an increase by a factor of 4 in resistivity when the input power changes from $3 \mu \mathrm{W}$ above $120 \mu \mathrm{W}$. 


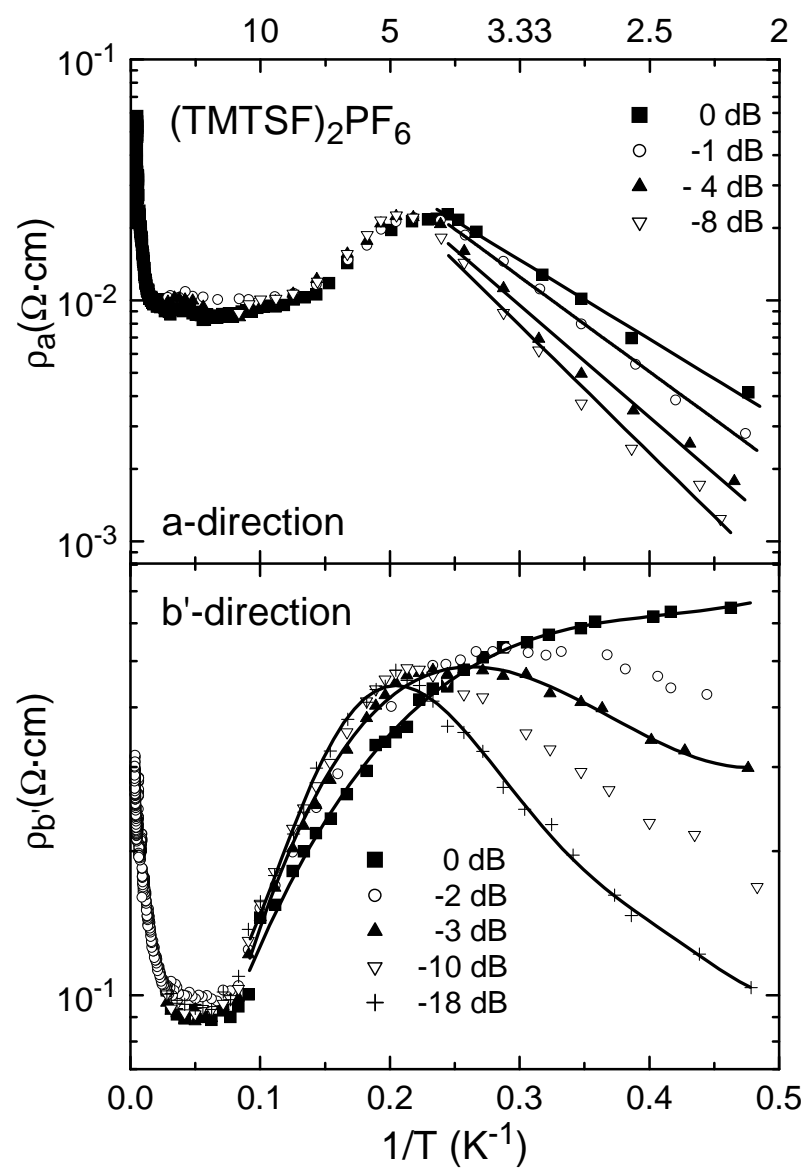

Fig. 6. Resistivity of (TMTSF $)_{2} \mathrm{PF}_{6}$ as a function of inverse temperature (Arrhenius representation) for different attenuation of the applied microwave power as indicated, leading to different strength of the microwave electric field inside the cavity. The experiments are performed at $33.5 \mathrm{GHz}$ with the electric field oriented (a) parallel and (b) perpendicular to the chain direction.

For a better understanding of the relation between SDW response and ac signal amplitude, frequency dependent conductivity measurements along the $a$-direction were performed in the radio frequency range $(20 \mathrm{~Hz}$ to $1 \mathrm{MHz}$ ) at different temperatures by changing the amplitude of the ac voltage $V_{S}$. A deviation from the linear behavior is observed if the applied ac signal amplitude $V_{S}$ increases above a certain threshold voltage $V_{S}^{\max }$ which also strongly depends on temperature (see Fig. 8 ).

The upper and lower panels of Fig. 9 show the real and imaginary parts of the dielectric constant as a function of frequency measured at $T=3 \mathrm{~K}$ for low $\left(V_{S}<V_{S}^{\max }\right)$ and high $\left(V_{S}>V_{S}^{\max }\right)$ ac amplitudes, respectively. While the observed dielectric response for very low ac fields was successfully fitted by a single HN mode of Eq. (1), similar attempts (dashed lines) to fit the data measured for higher fields failed. Consequently we tried to describe the data by the sum of two HN modes and achieved an excellent fit. The full lines in Fig. 9 correspond to the calculated fits to one (upper panel), or sum of the two HN functions (lower panel). A striking new result is that in addition to the

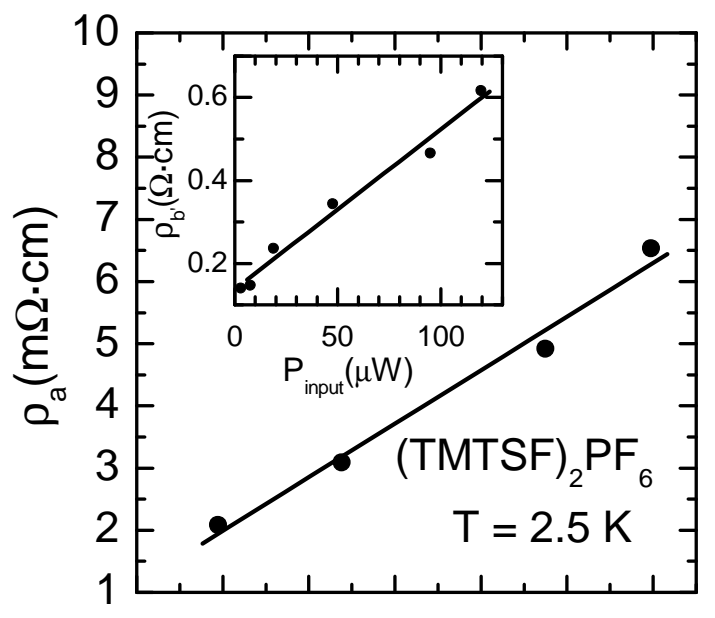

(TMTSF) ${ }_{2} \mathrm{PF}_{6}$

Fig. ' samples MO\&OM:

of $(\mathrm{T}$ XI'94. voltconts

into $\mathrm{t}$.

in the

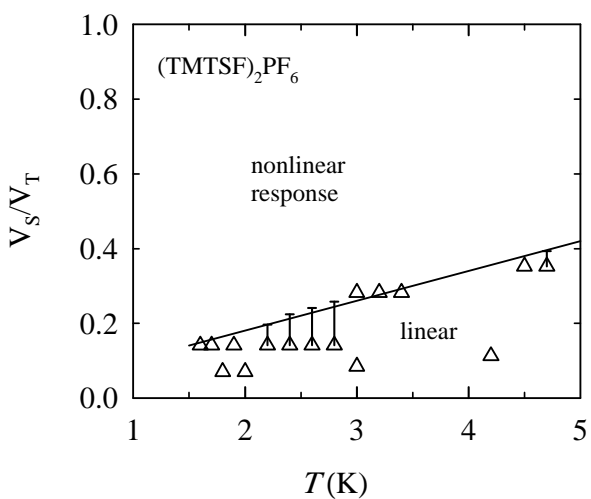

Fig. 8. ac-signal amplitude $V_{S}$ normalized to the dc threshold $V_{T}$, vs. temperbot

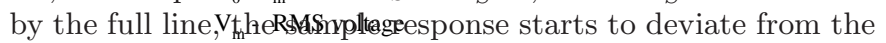
linear behaviory ${ }_{0}$ - ac field amplitude

already reported mode with $\Delta \varepsilon$ of the order of $10^{9}$, there is a mode centered at lower frequencies with one order of magnitude larger strength $\Delta \varepsilon \approx 10^{10}$ which occurs only at temperatures lower than $4 \mathrm{~K}$.

The dielectric strength $\Delta \varepsilon$ and the mean relaxation time $\tau_{0}$ of these two modes are plotted in Fig. 10] as a function of inverse temperature. The parameter $1-\alpha$, which describes the symmetric broadening of the relaxation time distribution function, is temperatures independent and similar for both modes: $1-\alpha=0.85$. Triangles (open and full symbols for low and high ac fields, respectively) stand for the mode already reported previously, and full squares (for high ac fields) stand for the new mode observed in this study for the first time. We will refer to these modes as high frequency (HF) and low frequency (LF) mode, respectively. 


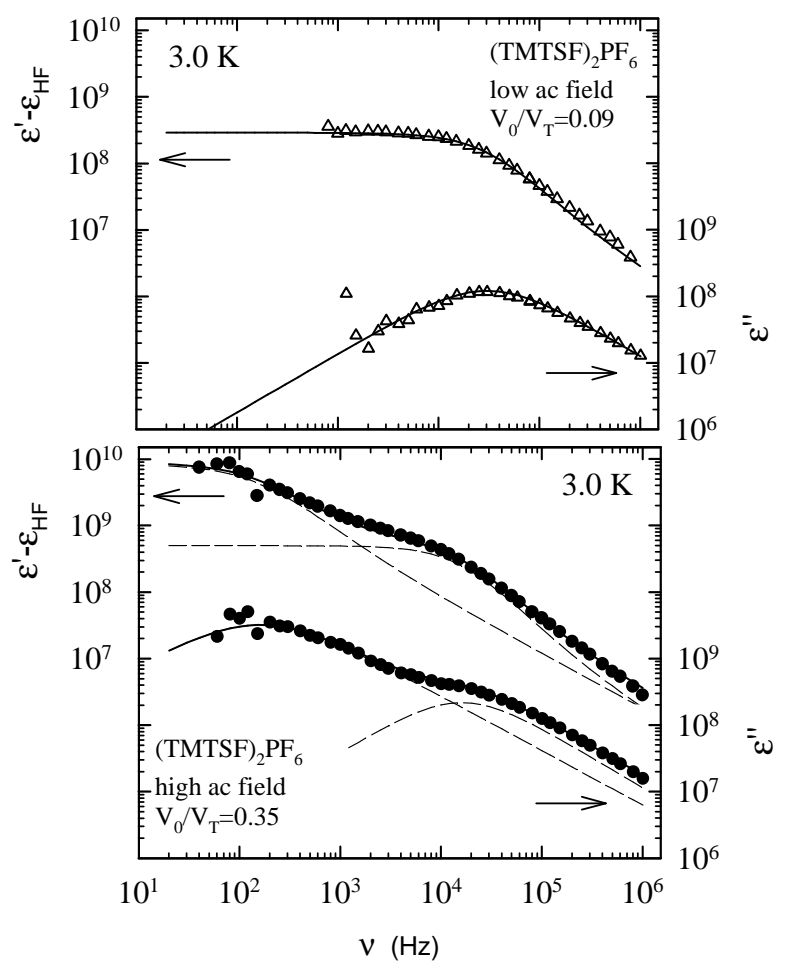

Fig. 9. Double logarithmic plot of the frequency dependenc of the real and imaginary parts of the dielectric function a $T=3 \mathrm{~K}$ for low (upper panel) and high (lower panel) ac am plitudes. The full lines are fits to the HN forms; the dasher lines represent the single $\mathrm{HN}$ form.

There are two effects induced by ac amplitudes $V_{S}$ larger than $V_{S}^{\max }$ (see Fig. 8): First, the number of lowfrequency relaxations increases, which effectively shifts the SDW loss peak to lower frequencies. Such an effect was previously observed for charge density waves and attributed to long-time relaxations from metastable states far from equilibrium in which the charge density wave is brought by large ac-signal amplitudes [27. Second, an additional mode appears below $4 \mathrm{~K}$, with much longer and temperature-independent mean relaxation time $\tau_{0} \approx 10^{-3} \mathrm{~s}$. At temperatures lower than $2 \mathrm{~K}$, the LF mode converges to the $\mathrm{HF}$ one, so that below $1.7 \mathrm{~K}$ only one mode can be resolved (Fig. 10).

\section{Discussion}

\subsection{Anisotropy of the Collective SDW Response}

The significantly reduced values of the activation energy found in the microwave resistivity right below the SDW transition at $T_{\mathrm{SDW}}$ for the $a$ and $b^{\prime}$ directions compared to dc data infer a strong frequency dependent response which is associated with the collective mode contribution to the electrical transport. It can be seen in both orientations, $a$ and $b^{\prime}$, but not in the $c^{*}$ direction.

It is worthwhile to consider the entire spectrum of the SDW response as suggested by G. Grüner and collabo-

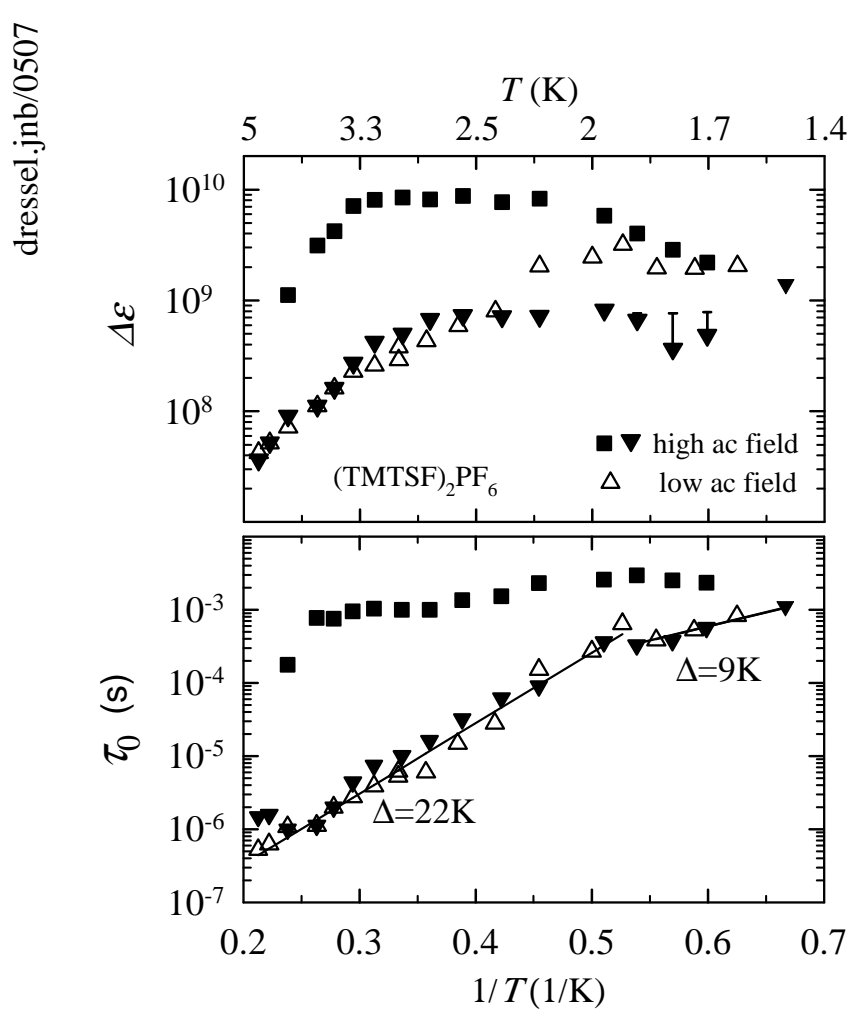

Fig. 10. Relaxation strength (upper panel) and mean relaxation time (lower panel) vs. inverse temperature. Triangles and squares for HF and the LF relaxation mode, respectively. Open and full symbols for low and high ac fields, respectively.

rators 3.7.19] (Fig. 10) Based on an extensive microwave study along the chain direction, it was proposed [7] that due to impurity pinning the collective SDW response in (TMTSF $)_{2} \mathrm{PF}_{6}$ is located around $5 \mathrm{GHz}$. At first glance, the conductivity below the energy gap decreases exponentially with decreasing temperature, except in the range of the pinned mode. As can be seen from Fig. [1 the present microwave experiments are performed in the range where the collective mode is still very pronounced, i.e. on the high-frequency shoulder of the pinned mode resonance. Hence the temperature dependent microwave conductivity is caused by two opposing effects: (i) the exponential freeze-out of the background conductivity caused by the uncondensed conduction electrons, and (ii) the build-up of the collective contribution. It was suggested that this mode does not gain much spectral weight as the temperature decreases, but the width and center frequency changes slightly [7. As discussed in Ref. [20 the presence of the enhanced microwave conductivity not only along the chains, but also perpendicular to them implies that the pinned mode resonance is present in the $b^{\prime}$ direction in a very similar manner compared to the $a$ axis. Hence the sliding density wave has to be considered a twodimensional phenomenon.

These results can be explained by looking at the actual Fermi surface of (TMTSF) ${ }_{2} \mathrm{PF}_{6}$ which is not strictly onedimensional but shows a warping in the direction of $k_{b}$ (and much less in $k_{c}$ ). From NMR experiments [4 it is known that the SDW corresponds to a wavevector $\mathbf{Q}=$ 
$\left[0.5 a^{*}, 0.24 b^{*},-0.06 c^{*}\right]$; which is slightly incommensurate with the underlying lattice along the $b^{*}$. Most important in this context, there is a four-fold periodicity in the real space not only along the best conductivity $a$-axis, but also perpendicular to it, i.e. along the $b$-axis. The tilt of the nesting vector is responsible for the similar collective SDW response found in the microwave experiments along the $a$ and $b^{\prime}$ directions.

\subsection{Field Dependence of the Collective SDW Response}

It is more difficult to explain the anomalous microwave response at even lower temperatures $(T<4 \mathrm{~K})$. The pinned SDW mode shifts to higher frequencies as the temperature decreases [7] and this can even lead to a decrease of the resistivity at certain frequencies. The non-monotonic behavior is therefore due to the decreasing single particle contribution while at the same time the pinned mode shifts into the relevant frequency window. Increasing the electric field makes this effect smaller since the pinned mode might remain at lower frequencies. The spectral weight is likely to be conserved. The effect can be readily understood in the simple picture of the washboard potential [3] which becomes anharmonic for larger driving force. In the real material, the impurity pinning centers are randomly distributed implying a distribution of metastable states around the equilibrium. This is reflected in the inhomogenously broadened resonances of the pinned mode, and in the broad loss peak in the radio-frequency range. Large ac electric fields, but still smaller than dc threshold for sliding, can drive the SDW into metastable states with longer relaxation times far from equilibrium configuration. As a result, the screened loss peak of the SDW shifts to lower frequencies. Similar effect can happen to the pinned SDW mode in the case of high frequencies. In particular the ac threshold field for non-linear response is strongly temperature dependent; hence at low enough temperatures the electric field applied to the sample inside the cavity could be high enough to exceed $E_{T}$ for ac fields. The sample dependence is caused by variations of the impurity concentration which act as pinning centers.

The deviation from the activated response in the microwave range sets in pretty abruptly below $T=4 \mathrm{~K}$, where in the radio-frequency range the LF mode appears. The relaxation of this LF mode is governed by very low energy barriers of the order of a few Kelvin in contrast to the HF process whose relaxation is governed by the singleparticle gap as it is the case for the dc conductivity. While the over-all behavior of the $\mathrm{HF}$ mode can be ascribed to the screened phason excitations, the spatial variations of the SDW, which is active in the LF process, should be restricted to rather narrow localized regions, so that the associated excitations (like solitons or domain walls) cannot contribute substantially to the dc conductivity. In addition, the almost temperature-independent mean relaxation time indicates that the free-carrier screening, whose influence remains to be visible in the $\mathrm{HF}$ process is insignificant for the LF process. This also implies that the relaxation entities in the LF process are much more localized in comparison to the entities responsible for the HT process 17,28. Discommensurations separating randomly distributed domains of $N=4 \mathrm{SDW}$, seem to be the most plausible candidate. Furthermore, the temperature behavior of the dielectric strength associated with the LF process indicates that the mode gains the full spectral weight at about $T=3.3 \mathrm{~K}$, and starts to diminish again below $2 \mathrm{~K}$ merging eventually into the $\mathrm{HF}$ mode below about $1.6 \mathrm{~K}$.

The observed dielectric and microwave responses seem to be manifestations of the same phenomenon: i.e. the transition from an incommensurate to a commensurate spin density wave, as suggested by NMR experiments 12 13. From the relaxation rate $1 / T_{1}$ of ${ }^{1} H$-NMR Takahashi et al. 13 constructed a phase diagram with three SDW subphases separated at $3.5 \mathrm{~K}$ and $1.8 \mathrm{~K}$ at ambient pressure. Note that these temperatures of the second SDW subphase define the region for which the LF mode is dominant when the ac-amplitude $V_{S}$ exceeds $V_{S}^{\max }=$ $0.1-0.3 V_{T}$.

\section{Conclusions}

The dc and microwave conductivity in the SDW state of (TMTSF $)_{2} \mathrm{PF}_{6}$ single crystals was measured along all three directions, $a, b^{\prime}$, and $c^{*}$. Deviations of the microwave response from the dc activation energy indicate that the pinned mode resonance is present in the $a$ and $b^{\prime}$ axes response, but not along the least conducting $c^{*}$ direction. The behavior can be explained by the nesting properties of the quasi one-dimensional conductor.

In addition, the electric-field dependence of the collective transport was studied in the radiofrequency and microwave range. The SDW response is non-linear above an ac threshold field because the SDW is driven into metastable states with longer relaxation times; this threshold is strongly reduced at temperatures below $4 \mathrm{~K}$. In the non-linear regime, radio-frequency dielectric measurements have identified, for the first time, two length and time scales involved in the low-temperature dynamics of the spin-densitywave state of (TMTSF) ${ }_{2} \mathrm{PF}_{6}$. In addition to the well known screened phason relaxation with Arrhenius-like decay determined by single-particle gap, there is an additional mode at lower frequencies with a temperature-independent relaxation time found at temperatures between $4 \mathrm{~K}$ and $2 \mathrm{~K}$. We ascribe this mode to discommensurations, that is, to short-wavelength excitations of the SDW close to commensurability. With increasing ac field, the screened phason loss-peak and the pinned-mode resonance shift to lower frequencies due to increasing contributions of longtime processes. From our microwave experiments we conclude that this behavior occurs in the $a$ and $b^{\prime}$ directions. Finally, below $T=1.7 \mathrm{~K}$, only one screened phason-like loss-peak can be detected whose relaxation is governed by low energy barriers of about half single-particle gap. 
P. Zornoza et al.: Anisotropic and field-dependent pinning of SDW

\section{Acknowledgment}

We thank Gabriele Untereiner for the crystal growth and sample preparation. The work was supported by the Deutsche Forschungsgemeinschaft (DFG) and Croatian Ministry of Science.

\section{References}

1. D. Jérome and H.J. Schulz, Adv. Phys. 31, 299 (1982).

2. G. Grüner, Rev. Mod. Phys. 66, 1 (1994).

3. G. Grüner, Density Waves in Solids (Addison-Wesley, Reading, 1994).

4. T. Takahashi, Y. Maniwa, H. Kawamura, and G. Saito, J. Phys. Soc. Jpn. 55, 1364 (1986).

5. L. Degiorgi, M. Dressel, A. Schwartz, B. Alavi, and G. Grüner, Phys. Rev. Lett. 76, 3838 (1996); M. Dressel, L. Degiorgi, J. Brinkmann, A. Schwartz, and G. Grüner, Physica B 230-232, 1008 (1997); V. Vescoli, L. Degiorgi, M. Dressel, A. Schwartz, W. Henderson, B. Alavi, G. Grüner, J. Brinkmann, and A. Virosztek, Phys. Rev. B 60, 8019 (1999).

6. G. Mihály, A. Virosztek, and G. Grüner, Phys. Rev. B 55, 13456 (1997).

7. S. Donovan, M. Dressel, Y. Kim, L. Degiorgi, G. Grüner, and W. Wonneberger, Phys. Rev. B 49, 3363 (1994).

8. S.Tomić, J.R.Cooper, D.Jérome and K.Bechgaard, Phys.Rev.Lett.62, 462 (1989); W. Kang, S. Tomić, and D. Jérome, Phys. Rev. B 43, 1264 (1991); S. Tomić, J.R. Cooper, W. Kang, D. Jérome, and K. Maki, J. Phys. I (France) 1, 1603 (1991).

9. G. Mihály, Y. Kim, and G. Grüner, Phys. Rev. Lett. 67, 2713 (1991); ibid. 66, 2806 (1991); O. Trætteberg, G. Kriza, and G. Mihály, Phys. Rev. B 45, 8795 (1992); F. Zámborszky, G. Szeghy, G. Abdussalam, G. Mihály, Phys. Rev. B 60, 4414 (1999).

10. K. Nomura, T. Shimizu, K. Ichimura, T. Sambongi, M. Tokumoto, H. Anzai, and N. Kigoshita, Solid State Commun. 72, 1123 (1989); M.Basletić, N.Biškup, B.KorinHamzić, A.Hamzić and S.Tomić, Fizika A 8, 293-310 (2000).

11. G. Kriza, G. Quirion, O. Trætteberg, W. Kang, and D. Jérome, Phys. Rev. Lett. 66, 1922 (1991).

12. W.G. Clark, M.E. Hanson, W.H. Wong, and B. Alavi, J. Phys. IV. (France) 3, C2 235 (1993); Physica B 194-196, 285 (1994); W.H. Wong, M.E. Hanson, B. Alavi, W. G. Clark, and W. A. Hines, Phys. Rev. Lett. 70, 1882 (1993); W.H. Wong, M.E. Hanson, W.G. Clark, B. Alavi, and G. Grüner, Phys. Rev. Lett. 72, 2640 (1994).

13. T. Takahashi, Y. Maniwa, H. Kawamura, K. Murata, and G. Saito, Synth. Met. 19, 225 (1987); T. Takahashi, T. Harada, Y. Kobayashi, K. Kanoda, K. Suzuki, K. Murata, and G. Saito, Synth. Met. 41, 3985 (1991).

14. J. Odin, J.C. Lasjaunias, K. Biljaković, P. Monceau and K. Bechgaard, Solid State Comm. 91, 523 (1994).

15. M. Basletic, B. Korin-Hamzic and K. Maki, Phys. Rev. B 65, 235117 (2002).

16. M. Dressel, K. Petukhov, B. Salameh, P. Zornoza, and T. Giamarchi, Phys. Rev. B (in press 2005); cond-mat/0409322

17. M.Pinterić, T.Vuletić, S.Tomić and J.U.von Schütz, Eur. Phys. J. B 22, 335 (2001).
18. O. Klein, S. Donovan, M. Dressel, and G. Grüner, Int. J. Infrared and Millimeter Waves, 14, 2423 (1993); S. Donovan, M. Dressel, O. Klein, K. Holczer, and G. Grüner, Int. J. Infrared and Millimeter Waves, 14, 2459 (1993); M. Dressel, O. Klein, S. Donovan, and G. Grüner, Int. J. Infrared and Millimeter Waves, 14, 2489 (1993).

19. M. Dressel and G. Grüner, Electrodynamics of Solids (Cambridge University Press, Cambridge, 2002).

20. K. Petukhov and M. Dressel, Phys. Rev. B (in press 2005); cond-mat/0408382

21. Note, the microwave data displayed in Fig. 3 4 and 6]are taken at different samples; their absolute values of resistivity vary. For microwave experiments it is also known that the absolute values also contain a large uncertainty due to the geometry (depolarization factor) [18.

22. W.M. Walsh Jr., F. Wudl, G.A. Thomas, D. Nalewajek, J.J. Hauser, P.A. Lee, and T. Poehler, Phys. Rev. Lett. 45, 829 (1980).

23. A. Jànossy, M. Hardiman, and G. Grüner, Solid State Commun. 46, 21 (1983).

24. L.I. Buravov, V.N. Laukhin, and A.G. Khomenko, Sov. Phys. JETP 61, 1292 (1985).

25. H.H.S. Javadi, S. Sridhar, G. Grüner, L. Chiang, and F. Wudl, Phys. Rev. Lett. 55, 1216 (1985).

26. The only exception is the $3 \mathrm{GHz}$ measurement, which was performed in a split ring resonator and not a enclosed cavity.

27. R.J.Cava, R.M. Flemming, R.G. Dunn, E.A. Rietman, and L.F. Schneemeyer, Phys. Rev. B 30, 7290 (1984).

28. M. Pinterić, M. Miljak, N. Biškup, O. Milat, I. Aviani, S. Tomić, D. Schweitzer, W. Strunz and I. Heinen, Eur. Phys. J. B 11, 217 (1999). 